\title{
Il medico di medicina generale e la nefrolitiasi
}

\section{E. Croppi}

Appartenenza

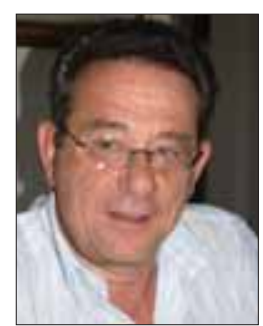

I 1 medico di medicina generale viene a conoscenza della malattia litiasica in una serie di circostanze che possono essere cosi riassunte:

1) come risultato di esami radiologici e/o ecografici eseguiti per la presenza o nel sospetto di altre malattie;

2) come risultato di esami radiologici e/o ecografici eseguiti dopo una colica risoltasi spontaneamente o dopo somministrazione di farmaci per la quale vi fosse il sospetto di colica renale;

3 ) come risultato di esami eseguiti durante un ricovero effettuato per la presenza di coliche non risoltesi né spontaneamente nè farmacologicamente;

4) dalla raccolta dell' anamnesi.

Al momento attuale, in gran parte di questi casi la figura professionale di riferimento è lo specialista urologo a cui il paziente afferisce o perché inviato dal medico del pronto soccorso, o perché inviato dal medico curante o per iniziativa personale. Solo nei casi di nefrolitiasi plurirecidivante o nel sospetto di una calcolosi secondaria vengono coinvolte altre figure professionali quali il nefrologo, l'endocrinologo, il radiologo i quali sovente in modo indipendente l'uno dall'altro e in tempi diversi valutano il paziente nell'ambito della propria specifica branca specialistica, spesso senza aver contatti fra di loro.

Il risultato di tutto questo è che al medico di medicina generale giungono spesso prescrizioni specialistiche discordanti provenienti da una o più branche specialistiche oppure come sovente accade il paziente viene dimesso dall'ambito ospedaliero con la sola prescrizione di aumentare l'introito idrico e sottoporsi a periodici controlli ecografici.

Come noto negli ultimi anni sono stati compiuti notevoli progressi per quanto attiene la terapia chirurgica della nefrolitiasi, infatti la varie tecniche di nefrolitotomia percutanea e la litotrissia extracorporea (ESWL) hanno in gran parte ridotto la necessità dell'intervento chirurgico tradizionale anche se non hanno ridotto il numero delle recidive e non sono sempre attuabili (1-3). Nonostante questi suc- cessi della chirurgia un corretto approccio medico alla malattia litiasica deve necessariamente affiancarsi a quello chirurgico al fine di individuare e correggere eventuali difetti metabolici, dietetici o semplicememente comportamentali alla base della nefrolitiasi e delle sue recidive (4). Le ricerche degli ultimi venti anni e l'affinarsi delle tecniche laboratoristiche hanno infatti consentito di individuare una serie di errori metabolici sensibili a fattori genetici e/o ambientali il cui squilibrio favorisce l'insorgenza della nefrolitiasi e la cui conoscenza appare indispensabile al corretto inquadramento della malattia e alla sua profilassi e terapia (5-10).

Fra tutte le influenze ambientali la dieta si è dimostrata di particolare importanza e recenti ricerche hanno consentito di mettere a fuoco i principali fattori dietetici coinvolti nella malattia e di sfatare luoghi comuni appartenenti al passato $(11,12)$.

Un altro importante aspetto della malattia litiasica, anche questo frutto della ricerca degli ultimi anni, è il coinvolgimento del metabolismo osseo emerso con l'affinamento delle 


\section{Giornale \\ di Tecniche \\ Nefrologiche \\ \& Dialitiche \\ Anno XVI n ${ }^{\circ} 3$ \\ (C) Wichtig Editore 2004

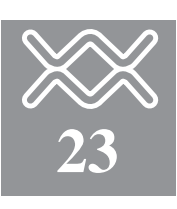

tecniche densitometriche e di dosaggio dei marker di turn-over osseo (1315 ).

Nell'ambito di questo complesso quadro che comprende aspetti diagnostico-terapeutici, ma anche di ricerca epidemiologica e clinica quale è attualmente e quale potrebbe essere in futuro il ruolo del medico di medicina generale? La mia opinione al riguardo è che il medico di medicina generale non sia adeguatamente informato sull'argomento per la scarsità di corsi di aggiornamento sul tema, di conseguenza anche le possibilità attuali di coinvolgerlo direttamente sono alquanto scarse.

$\mathrm{E}$ auspicabile pertanto un maggior impegno nella formazione del medico di medicina generale per quanto attiene la nefrolitiasi non solo perché è una malattia con una prevalenza del 4\%-10\% (16) ma anche perché è una forma morbosa che si pone al crocevia fra varie branche specialistiche per cui vi possono spesso essere dubbi e difficoltà interpretative sulle varie indicazioni provenienti dai vari settori specialistici.

La mia opinione è che, dopo un adeguato aggiornamento, il ruolo del medico di medicina generale potrebbe essere il seguente:

- Partecipazione attiva a studi di carattere epidemiologico

- Partecipazione a uno screening di primo livello che consenta di individuare forme di calcolosi secondaria

- Monitoraggio della malattia e follow-up

- Ruolo di collegamento tra la varie branche specialistiche

- Evidenziare nei propri pazienti eventuali fattori di rischio per la malattia.

In conclusione penso alla nefrolitiasi come a una malattia sempre più multidisciplinare per cui non credo che in futuro esisterà un determinato settore specialistico per la valutazione medica del paziente litiasico, ma credo vi saranno figure professionali provenienti da varie branche specialistiche che matureranno esperienza sulla materia e di conseguenza saranno le figure professionali di riferimento per il medico di medicina generale e per

\section{BIBLIOGRAFIA}

1. Chaussy C, Schmiedt E, Jocham D, Brendel W, Forssmann B, Walter V. First clinical experience with extracorporeal induced destruction of stones by shock waves. J Urol 1982; 27: 417-20.

2. Fuchs GJ, Miller K, Rassweiler J, Eisemberger F. Extracorporeal shock wave lithotripsy: one year'sexperience with the Dornier lithotripter. Eur Urol 1985; 11: 145-9.

3.

Putman SS, Hamilton BD, Johnson DB. The use of shock wave lithotripsy for renal calculi. Curr Opin Urol 2004; 14: 117-21.

4. Consensus Conference. Prevention and treatment of kidney stones. JAMA 1988; 260: 978-81.

5.

Pak CYC. Phisiological basis for absorbtive and renal hypercalciurias. Am J Physiol 1979; 237: F41523.

6. Marangella M, Fruttero B, Bruno $M$, Linari F. Hyperoxaluria in idiopathic calcium stone disease: further evidence of intestinal hyperabsortion of oxalate. Clin Sci 1982; 63: 381-5.

7. Vezzoli G, Caumo A, Baragetti I, et al. Study of calcium metabolism in idiopathic hypercalciuria by strontium loading test. Clin Chem 1999; 45: 257-61.

8. Baggio B, Gambaro G, Favaro S, Borsatti A. Prevalence of hyperoxaluria in idiopathic calcium oxalate kidney stone disease. Nephron 1983; 35: 11-4.

9.

Croppi E, Vitale C, Bevilacqua $\mathrm{M}$, et al. Consensus statement on diagnosis of primary hypercalciuria. Clinical Cases in Mineral and Bone Metabolism 2004; 1: 73-5.

10. Marangella M. Metabolic evaluation of calcium nephrolithiasis. J Nephrol 1995; 8: 179-84

11. Borghi L, Schianchi T, Meschi
T, et al. Comparison of two diets for the prevention of recurrent stones in idiopathic hypercalciuria. N Engl J Med 2002; 346: 77-84.

12. Curhan G, Willett W, Speizer F, Spiegelmann D, Stampfer M. Comparison of dietary calcium with supplemental calcium and other nutrients as factors affecting the risk for kidney stones in women. Ann Intern Med 1997; 126: 497-504.

13. Jaeger P, Lippuner K, Casez JP, Hess B, Ackermann D, Hung C. Low bone mass in idiopathic renal stone formers: magnitude and significance. J Bone Miner Res 1994; 9: 1525-32.

14. Caudarella R, Vescini F, Buffa A, Stefoni S. Citrate and mineral metabolism. Front Bio Sci 2003; 8 (suppl): S1084-106.

15. Vitale C, Marangella M, Isaia GC, Petrarulo M, Rovera L, Ramello A. The effects of alendronate on urine biochemistry and mineral metabolism in stone formers with fasting hypercalciuria. It J Min Electr Metab 1998; 12: 5-8.

16. Trinchieri A. Epidemiologia della calcolosi renale. Atti del Convegno sulla Calcolosi Renale, Chianciano Terme, 14-15 Aprile, 2000. 